\title{
Resource Provider Selection for Personal Live Content Delivery in User-Provided Platform
}

\author{
Joseph C. H. So and P. C. Wong
}

\begin{abstract}
The personal live content delivery is useful in many different application scenarios. Providing this service using end user provided network resource is a feasible solution with the advantage of fast deployment. However, in order to provider some service level agreement, the platform has to acquire sufficient network resources through resource acquisition, while, to ensure the provision, the corresponding resource providers are remunerated. For the perspective of service operators, selecting providers that effectively fulfilling SLA and efficiently reducing costs are their primary goals. We illustrate the feasibility of deploying a local search heuristic algorithm, Tabu search, in searching resource providers. Our simulations showed that their effectiveness in satisfying the commitment on the resource provision stated in SLA and minimizing the related costs. We find that the systematic choice of initial solution can improve the effectiveness of Tabu Search.
\end{abstract}

Index Terms-Peer-to-peer network, resource acquisition, content distribution, tabu search.

\section{INTRODUCTION}

"Broadcast Yourself" is the slogan used by Youtube [1], a very popular video sharing platform. Everyone can upload his/her self-made videos to the Youtube platform and make them available to the public. Youtube is platform for personal content delivery, in which individual users provide their own content and rely on the platform to perform content delivery.

The content broadcasters can publicize their streaming contents and rely on Youtube for the platform provision. However, they has to follow the rules defined by Youtube. The video format, the resolution, the frame rate, the video length and its size are restricted by the service operator. These restrictions are understandable from the view of platform management, as the service platform is pre-installed. Limited varieties will relieve the burden of management and the uncertainty in server resource consumption, as installation of servers requires long time for planning.

Service provided by Youtube uses the traditional client/server model. It needs tremendous server spaces as well as outgoing bandwidth from the server platform to cater for the growth of channel number.

A recent survey by Stream Media.com [2] shows that the price and the geographic reach of network are among the top three concerns of content delivery services. A particular content broadcaster would expect the servers be located close to the target viewers, so that it can deliver the data timely.

Manuscript received September 15, 2012; revised November 30, 2012.

Joseph C. H. So is with the the Hong Kong Polytechnic University, Hong Kong, China (e-mail:joseph.so@hkcc-polyu.edu.hk).

P. C. Wong is with the Hang Seng School of Commerce, Hong Kong, China (e-mail:pcwong@hsmc.edu.hk).
The need for end-user broadcasting inspires the introduction of personal live content delivery platform. This platform allows end-user renting the platform resource to deliver live content with user tailor-made specifications. We can take several scenarios that such application: (1) A content broadcaster situates in Hong Kong and wants his product promotion video broadcasted specific to Toronto with high availability in order to synergize the promotion campaign there. (2) In an international football semi-final match, naturally, people in two countries in a semi-final match are more interested than the other countries. This sudden demand is found only after knowing the quarter-final results a couple of days before. (3) A company is listed in two stock markets. A press conference releasing a sudden announcement has to be broadcasted to both places.

These cases share common requirements that such content delivery services need some instantaneous or short-term solutions and the service provision is confined to some specific areas. However, in the traditional content delivery network (e.g. Akamai [3]), servers are alreadly installed in some designated places. To cater for the above mentioned ad hoc service requirement, it requires installation of oversea servers, which is usually not financially viable and not timely, as cost of server installation is high and it requires a long deployment period. Furthermore, as quality of video is also controlled by the platform provider, it greatly limits the flexibility of content production.

Personal content delivery platform distinguishes from these systems by the number of content broadcasters being numerous. Furthermore, the content broadcasters would like to have better control on the channel quality. They want to manipulate not only the size and the length of the video, but also the availability and the playback latency. In other words, the quality of the channel is customized according to broadcasters' preferences.

The peer-to-peer (P2P) network gives a promising potential to support this type of "many-to-many" broadcast. The end users are providing the network resource for building the platform. However, in order to provide reliable services, the platform has to provide sufficient network resource to support the quality requirement of the users. In $\mathrm{P} 2 \mathrm{P}$ network, the guarantee of resource provision from individual end nodes is a great challenge as they can come and go freely. It requires a resource acquisition $(R A)$ strategy that supports the quality of service and is cost efficient.

In many previous works [4], [6] the significance of incentives and remuneration in service providing in $\mathrm{P} 2 \mathrm{P}$ networks are widely discussed. In our previous work [6], we have proposed a RA service model to analyze the cost involved in service provision. Such explicit remuneration 
strategies can facilitate the fulfillment of SLA in P2P based content distribution services. This paper discusses the problem of resource provider selection for personal live content delivery built by end-user platform. Service operators use the end-user provided to build the platform to allow content broadcasters to subscribe with a set of customized service level agreement (SLA) parameters. In particular, we focus on the resource provider selection strategy and its cost implication. We show that with an appropriate resource provider selection scheme, we can minimize the cost required to acquire resources to meet the SLA requirements of content broadcasters.

\section{SERVice Provision AND Resource AcQuisition}

Before introducing our resource provider selection scheme for personal broadcasting, we first review the model of service provision of personal live content delivery platform. We distinguish different parties in current service provision. Then, the requirement for the service quality is specified in the service level agreement is introduced.

Service Provision Model of Personal Live Streaming

In the service provision model (Fig. 1), there are three parties in a system: 1) Content Broadcasters (CB), 2) Service Operator (SO), and 3) viewers. Content broadcasters upload their contents on their personal channels to the platform provided by the service operator. The service operator will create indexes for the channels. When a watcher looks for a particular channel on the operator's platform, the channel is retrieved and the content is delivered. This model applies to both client/server-based and P2P-based platforms.

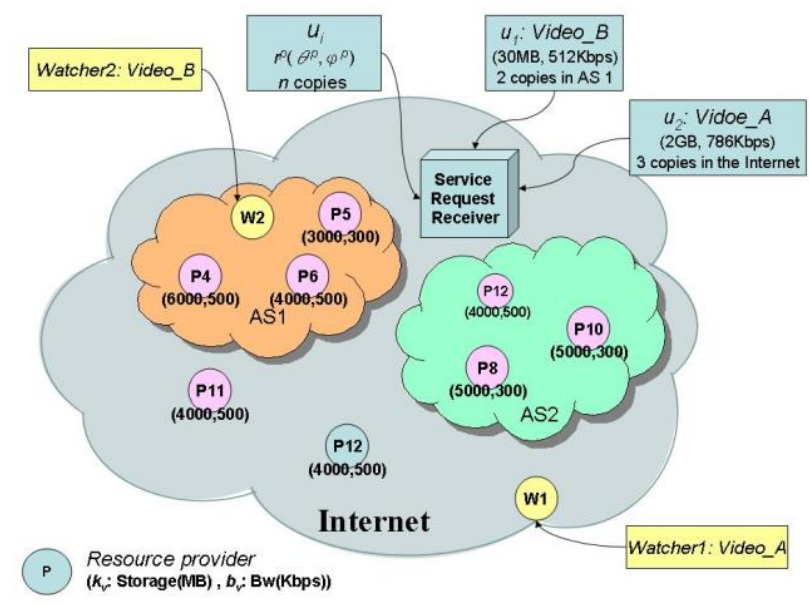

Fig. 1. Service provision for personal live streaming.

In a client/server-based system, the service platform is deployed by a set of servers owned by the service operator.

In a P2P system, the service platform is formed by the network resources provided the end-user nodes acting as resource providers $(R P)$ in the platform. Peers that have committed to contribute an amount of resources first register as resource providers (RP) (which can be easily implemented by software module installation). The providers are remunerated as the incentives for the commitment for the resource provision. When a content broadcaster (CB) registers for a RA service from the service operator (SO), $\mathrm{CB}$ specifies the streaming quality in SLA and SO will look for the available resources from resource providers. The available resources are unreserved portions of the resources that are committed by RPs. CB can request for special requirements on the guaranteed number of concurrent viewers in the domain (specified by the autonomous system (AS)) stated in SLA.

\section{A. Resource Acquisition and Overlay Formation for Live Streaming}

Content broadcaster subscriptions are specified with the expected service quality. The quality of service for a broadcast session is described in a set of metrics in SLA. We consider the guarantees in the number of viewers, their data rates and the viewers' location as the main SLA parameters provided by the RA services. The peers' uploading bandwidths are considered as the main resources. CB is also referred as a service user $(u)$ to reflect its role as a RA service user. Therefore, the requirement description vector $\left(R D V_{u}\right)$ becomes $\left(\phi_{u}, \omega_{\mathrm{u}}, D_{\mathrm{u}}\right)$, where $\phi_{u}$ is the bandwidth requirement of each stream for each viewer, $\omega_{\mathrm{u}}$ is the guaranteed number of viewers to be served and $D_{\mathrm{u}}$ is the domain of the target viewers situated (where a domain can be defined by groups of IP address or other parameters depending the user requirement).

Bandwidth usage among the intermediate nodes has to be considered when formulating the problem. The cost of service provision refers to the aggregate remuneration for services requiring bandwidth guarantee.

\section{PROBLEM ForMULATION}

With the above model, we consider the selection appropriate end nodes as resource providers, so that the user requirements are satisfied with the delivery tree formed. On the other hand, from SO's prospective, the cost involved in $\mathrm{RA}$ is the main concern.

\section{A. Revision on the RA Model}

We formulate the problem as following. Content broadcasters are users $U=\left\{u_{1}, u_{2}, \ldots\right\}$ and resource providers are $P=\left\{p_{1}, p_{2}, \ldots\right\}$ Each peer node $v$ has outgoing bandwidth $b_{v}$, describing its commitment on resources sharing. We treat the data stream as fluid, so it can be infinitely divided into any number of sub-streams as long as the total data download rate of all sub-streams is the same as the original one. $b_{v, u}$ defines the partition allocation of the uploading bandwidth to content broadcaster $u \in U$ in node $v \in P$.

\section{B. Problem Statement}

We aim at minimizing the cost of resource reservation while maintaining the amount of resources in the system capable of serving all users' requests. The research question being addressed is:

- How can the P2P resource providers be found without a centralized controlling agent so that all content broadcasters requirement description vectors, in terms of the required bandwidth, the guaranteed number of concurrent viewers in the target domain, are satisfied with minimum cost?

As the resources in each peer are limited, an efficient resource acquisition scheme is needed to determine the 
amount of resources that should be allocated to each user from each peer in the network.

Each content broadcaster is guaranteed with the bandwidth of $\phi_{u}$, to each of $\omega_{u}$ viewers in a domain $D_{u}$. The $R D V_{u}$ parameters become $\left(\phi_{u}, \omega_{u}, D u\right)$. We denote $b_{u}$ as the product of $\phi_{u}$, and $\omega_{\mathrm{u}}$ (i.e. $\phi_{u}, \times \omega_{\mathrm{u}}$ ). It is the resource demand of each content broadcaster. Hence, we target to derive the decision variables. $P_{u}$ is a set of registered resource providing nodes acting as the candidates of resource providers for $u$, and $V$ in $P_{u}$ can directly communicate with neighbouring peers and they can exchange their neighbours' availabilities. The sets of decision variables are defined as $\left\{b_{v, u}\right\}$, representing the representing the bandwidth reserved for each user $u$ on peer $v$ respectively.

Problem Statement becomes: Given a set of resource providers with cost per unit bandwidth $\left\{\mathrm{q}_{\mathrm{v}}(\mathrm{b})\right\}_{\mathrm{v} \in \mathrm{P}}$ and outgoing bandwidth $\left\{b_{v}\right\}_{v \in P}$ serving a set of users $U$, each with the requirement description vector $\left(\phi_{\mathrm{u}}, \omega_{\mathrm{u}}, \mathrm{D}_{\mathrm{u}}\right)$. Find a set of resource providing nodes $\left\{\mathrm{P}_{\mathrm{u}}\right\}_{\mathrm{u} \in \mathrm{U}}$ in domain $\mathrm{D}_{\mathrm{u}}$ and the resource reservation on the nodes $\left\{b_{u, v}\right\}_{v \in P}$ such that the network cost is minimized while all users' resource demand for all services are satisfied and $\mathrm{P}_{\mathrm{u}}$ form a content delivery tree.

Mathematically, the optimization problem can be formulated as finding the values of $b_{u, v}$ for the following objective function:

Minimize:

$$
Q=\sum_{u \in U} \sum_{v \in P_{u}}\left(b_{u, v} \cdot q_{v}\left(b_{u, v}\right)\right)
$$

Subject to:

$$
\begin{gathered}
w_{u} \leq \sum_{v \in P_{u}}\left\lfloor\frac{b_{u, v}}{\Phi_{u}}\right\rfloor-\left(\left|P_{u}\right|-1\right), \forall_{u} \in U, P_{u} \subseteq D_{u} \\
b_{v} \geq \sum_{u \in U} b_{u, v}, \forall_{v} \in P_{u}
\end{gathered}
$$

$q_{v}(b)$ can be a function of the unreserved bandwidth of a provider node and the load on that node. The constraint 2 is defined by the bandwidth requirement of each user in the delivery tree formation. A user guaranteed with $\omega_{\mathrm{u}}$ viewers each with $\phi_{u}$ bps. The maximum number of streams that can be served by a node $e_{u, v}=\left\lfloor\frac{b_{u, v}}{\Phi}\right\rfloor$. The number of viewers that can be served by a node is $\omega_{u, v}=e_{u, v}-O_{v}^{\#}(\mathrm{We}$ denote $O_{v}^{\#}$ and $I_{v}^{\#}$ as the number of streams outgoing from and incoming to $V$ connected to other nodes in the tree). We derive the number of streams in Lemma 3.1

Lemma 3.1: The maximum number of streams provided by $P_{u}$ is $\sum_{v \in P_{u}} e_{u, v}-\left(\left|P_{u}\right|-1\right)$.

Proof:

$$
\begin{aligned}
\max w_{u}= & \max \sum_{v \in P_{u}}\left(w_{u, v}\right) \\
& =\sum_{v \in P_{u}}\left(e_{u, v}-O_{v}^{\#}\right)=\sum_{v \in P_{u}} e_{u, v}-\sum_{v \in P_{u}} O_{v}^{\#} \\
& =\sum_{v \in P_{u}} e_{u, v}-\sum_{v \in P_{u}} I_{v}^{\#}=\sum_{v \in P_{u}} e_{u, v}-\left(\left|P_{u}\right|-1\right)
\end{aligned}
$$

The last step of the proof follows that the tree property that each outgoing node must have one and only one upstream provider excluding the source.

The constraint 3 guarantees the provider nodes with sufficient bandwidth to satisfy the requirement. It is also assumed that a domain has adequate amount of resources available for supporting the all user requirements, that is, $\sum_{u \in U} \Phi_{u} \leq \sum_{v \in P} b_{v}$

\section{TABU SEARCH FOR RP SELECTION}

To solve the above optimization, analytical constrained optimization (such as combinatorial optimization, Lagrangian optimization and dynamic programming) are techniques that aiming at globally optimal solution. The computation time required to solve these problems by analytical methods may grow exponentially with the size of the problems. In the context of networks consisting of more thousands of end-user provided nodes, the analytical methods are not feasible.

Tabu Search (TS) was originally proposed by Glover [7], and since then it has been extensively studied and applied to several optimization problems with great success. It is a heuristic searching approach widely used in assignment problems [8]. Although there is no proof for the convergence to the global optimal, it shows its practical use in many assignment problems [9], [10] in which our resource allocation problem fails.

The key steps involved in Tabu Search are: (i) Get initial solution; (ii) Move manager generates a series of moves; (iii) Evaluate moves by the objective function; (iv) Pick the best non-tabu move as the current solution; (v) Operates on the current solutions; and (vi) Go back to step (ii). TS manages a memory of solutions or moves recently applied, called tabu list. To prevent being trapped in a local optimum, when a local optimum is reached, the search carries on by selecting a candidate solution not as good as the current one. To avoid cyclic searching, TS discards the neighbouring candidates that have been previously applied such that the previous solutions will not be chosen again.

\section{A. Bandwidth-Guarantee Tabu Search Algorithm}

In the implementation, we develop a Bandwidth-Guarantee Tabu Search Algorithm (BGTS) to realize TS for our peer selection problem. We use tabu list in the peer selection. In each iteration, we create a list of moves as changes of the providers for a user. We use the recency-based tabu list. The tabu list records the recent changes in the providers for each user. It forbids a move if it contains either an item recently added or an item recently dropped. BGTS has the following definition in TS:

1) Objective function: Miniming Eqt.(1). 
2) Solution: The solution for each user $h_{s}$ is a vector storing the list of providers streams reserved for $u$. $h_{s}$ may contain duplicating elements. A provider serving $m$ streams for $u$ will have $m$ elements in $h_{s}$.

3) Neighbour: Given a solution $h_{s}$ containing a list of providers, its neighbour is a solution with one of the providers replaced by another.

4) Move: A move is a change in the solution. The move anager will create a set of candidate solutions $h_{c}$ from $h_{s}$ as neighbours. The provider stated in each element in $h_{s}$ is changed. For each move, a move value is evaluated to represent the change in the objective function value as a result of the move.

TS starts at a trivial initial solution and proceeds iteratively. TS methods operate under the assumption that a neighbourhood can be constructed to identify "adjacent solutions", or moves that can be reached from any current solution. In each iteration, a set of candidate moves is selected from the neighbourhood of the current optimal solution. The move with the lowest cost (i.e. the best value) is selected and applied to the current solution to generate a new solution. The Tabu List in TS contains a subset of the moves in the neighbourhood as forbidden moves. These moves are previously attempted, but usually attractive (due to its large improvement in the objective value). It reduces the chance of being trapped in the local minimum. Peer found in this tabu list are excluded from the candidate list. The search stops at a designated number of iterations or no improvement in the objective value after a number of iterations.

In the original proposed TS framework, there is no specification on how the initial solution should be generated. If a good initial solution is provided, the efficiency of optimization can be enhanced. We consider the provision of good initial solution. Therefore we propose a scheme for choosing the initial solution, called First Feasible Solution (FFS). By FFS, the initial solution will be able to give a set of providers so that the total bandwidth will be sufficient to satisfy the user's requirement according to Lemma 3.1. This FFS is implemented by Algorithm 1

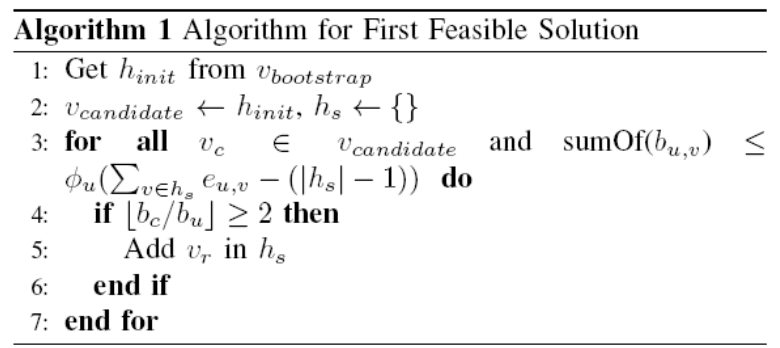

\section{Simulation Results On TS}

In order to evaluate the effectiveness of FFS, we compare it with the scheme that the initial solution is generated randomly regardless if it is feasible.

We implement a well-known Tabu Search Framework OpenTS [11]. OpenTS is a Java-based TS framework in object-oriented design. Without loss of generality, for illustration purpose, we choose the expression $q_{u}\left(b_{r, v}\right)=F_{b}+$ $G_{b}\left(b_{v}-b_{r, v}\right)$, where the reserved bandwidth in $v, F_{b}$ is a fixed unit costs of an instant of a storage reservation and a bandwidth reservation $G_{b}$ are the unit costs coefficient reflecting the bandwidth availability (The argument for such expression is discussed in [6]).

In the simulation, the network has 1000 nodes with average bandwidth $300 \mathrm{kbps}$ (in even distribution in range of $20 \%$ ). Users have average bandwidth request $\bar{\phi}_{u}^{*}$ and the guaranteed number of streams for each user is $2{\overline{\omega_{u}}}^{*}$. The number of iteration is set to be 5000 and the length of Tabu List is 7 (this has been found sufficient to prevent cycling [12] in the most cases). The users are added to the system one-by-one.

The results are shown in Fig. 2. We measure the effectiveness of the two approaches by two metrics: Ratio of Successful Provision of Streams and Cost of Successful Provision of Streams. We first set $\bar{\omega}_{u}$ as ${\overline{\omega_{u}}}^{*}$. As we can see from Fig. 2(a) and 2(b), under the given number of iterations in searching, the TS with FFS can significantly increase the successful rate of provider selection for the required resources, when the number of users is increasing. When we examine the expected costs for resource remuneration, we can see from Fig. 2(c) and 2(d) that the cost difference is within $5 \%$ which is not significant.

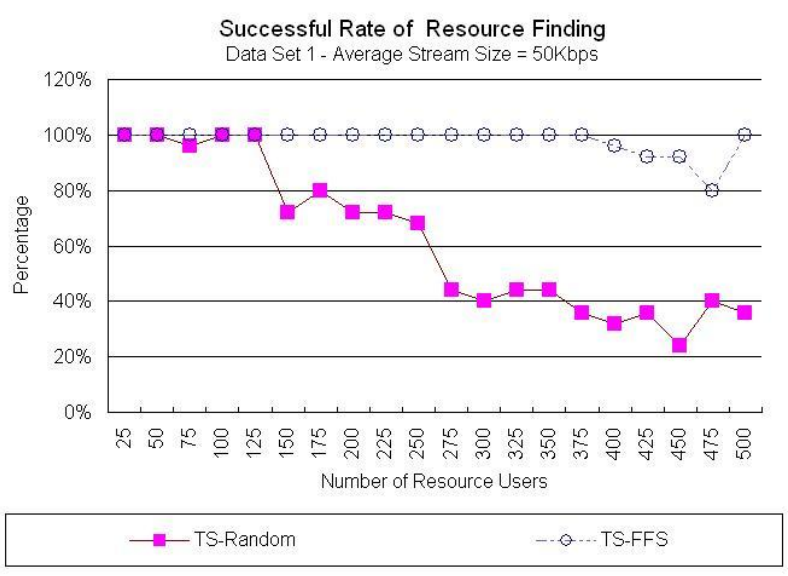

(a) Ratio of Successful Provision of streams $\left(\bar{\phi}_{u}=\bar{\phi}_{u}^{*}\right)$

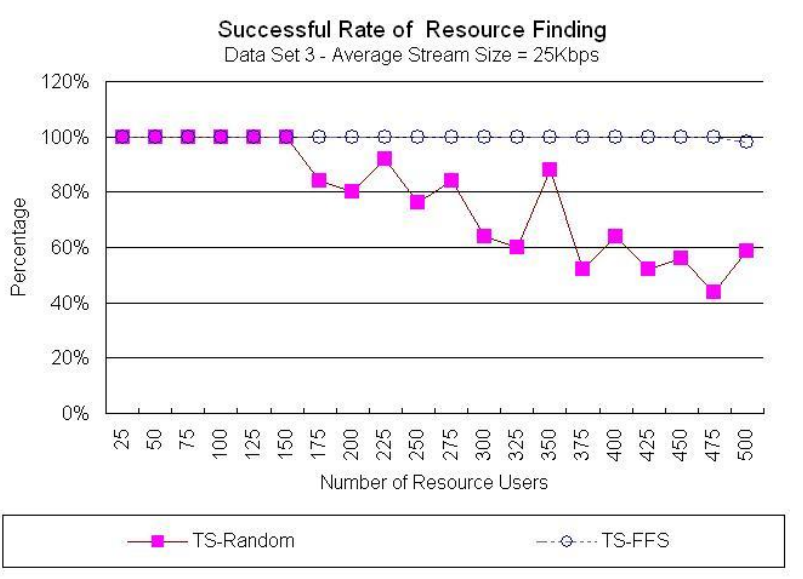

(b) Ratio of Successful Provision of streams $\left(\bar{\phi}_{u}=0.5^{\bar{\phi}_{u}}\right)$ 
Cost of Successful Provision

Data Set 1 - Average Stream Size $=50 \mathrm{Kbps}$

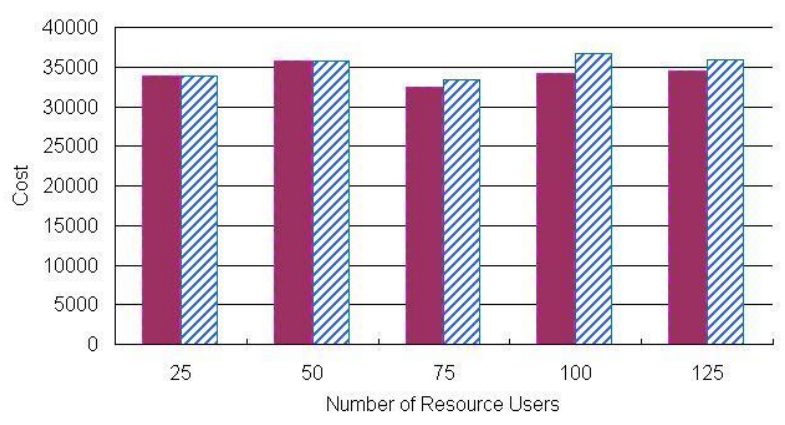

\begin{tabular}{l}
$\square$ TS-Random \\
$\square T S-F F S$ \\
\hline
\end{tabular}

(c) Cost of Successful Provision of streams $\left(\overline{\phi_{u}}=\bar{\phi}_{u}^{*}\right)$

Cost of Successful Provision

Data Set 3 - Average Stream Size $=25 \mathrm{Kbps}$

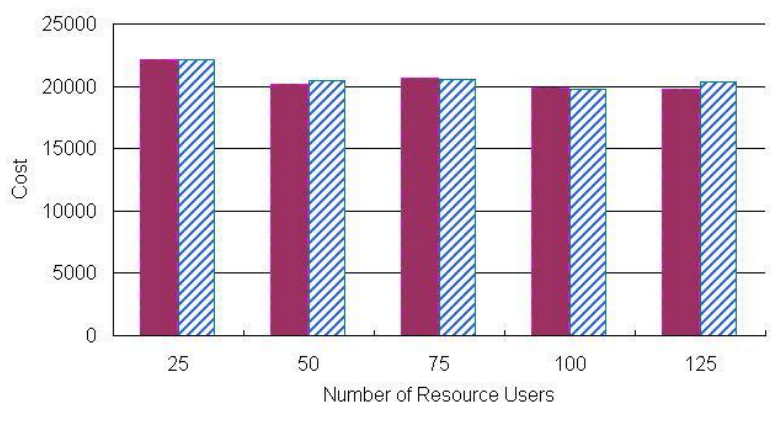

\begin{tabular}{|l|l|}
\hline TS-Random $\quad$ QTS-FFS \\
\hline
\end{tabular}

(d) Cost of Successful Provision of streams $\left(\overline{\phi_{u}}=0.5 \bar{\phi}_{u}^{*}\right)$

Fig. 2. Comparison of TS-random and TS-FFS. Network of 1000 nodes $\bar{b}$ $=300 \mathrm{kbps}$ in even distribution in range of 20\%) and $\bar{u}=10$ (in even distribution in range of 50\%) Number of iteration is 5000 and Tabu List length is $7 . F_{b}=100$ and $G_{b}=1$.

With First Feasible Solution (FFS), the BGTS can increase its capability in searching solution to fulfill the user requests, while the cost of provision is basically the same as TS with random initial solution.

\section{CONCLUSION}

We consider the resource acquisition model for platform acquiring user provided resources for supporting live data content delivery. We focus on the personal live streaming with the targeted number of viewers in an autonomous system. The SLA contains the bandwidth size and the minimum number of viewers in the customized targeted domain.

The objective is to satisfy the content broadcaster requirements with the minimized cost. We formulate an efficient provider selection strategy that is capable of satisfying the SLA in the content delivery network. Determining all the decision variables $\left(b_{u, v}\right)$ for each $u$ and $v$ simultaneously for a network with practical size is nearly impossible. Therefore, a heuristic approaches is deployed to generate near-optimal solutions. We implement Tabu Search and modify its initial solution formation to develop BGTS and show that the systematic choice of initial solution can improve the effectiveness of the optimization.

\section{REFERENCES}

[1] YouTube. (2010). [Online]. Available: http://www.youtube.com.

[2] CDN survey data: Price and customer service ranked most important. Mar 2008. [Online]. Available: http://blog.streamingmedia.com/the_business_of_online_vi/2008/03/c dn-survey-data.html.

[3] Streamingmedia. http://www.streamingmedia.com/.

[4] Akamai. (2008). http://www.akamai.com.

[5] G. Tan and S. A. Jarvis, "A payment-based incentive and service differentiation scheme for peer-to-peer streaming broadcast," IEEE Trans. Parallel Distrib. Syst., vol. 19, no. 7, pp. 940-953, 2008.

[6] G. Ruffo and R. Schifanella, "Fairpeers: Efficient profit sharing in fair peer-to-peer market places," J. Netw. Syst. Manage., vol. 15, no. 3, pp. 355-382, 2007.

[7] J. C. H. So and P. C. Wong, "Cost-aware resource acquisition for guaranteed service in $\mathrm{P} 2 \mathrm{P}$ networks," Services Part II, IEEE Congress on, vol. 0, pp. 183-188, 2008.

[8] F. Glover and M. Laguna, Tabu Search.1em plus $0.5 \mathrm{em}$ minus $0.4 \mathrm{em}$ Dordrecht, Netherlands: Kluwer, 1996.

[9] J. S. Kapov, "Extension of a Tabu search adaptation to the quadratic assignment problem," CAOR, vol. 21, pp. 855-865, 1994.

[10] H. R. Lourenco and D. Serra, "Adaptive search heuristics for the generalized assignment problem," Mathware and Soft Computing, vol. 9, pp. 209-234, 2002.

[11] N. Charbonneau and V. Vokkarane, "Tabu search meta-heuristic for static manycast routing and wavelength assignment over wavelength-routed optical wdm networks," in IEEE ICC.Cape Town, South Africa: IEEE, May 2010.

[12] 2plus 43minus 4OpenTS. Opents. Oct 2008. [Online]. Available: http://www.coin-or.org/Ots/. [Online]. Available: http://www.coin-or.org/Ots/index.html =0pt

[13] C. R. Reeves, Ed., Modern heuristic techniques for combinatorial problems.1em plus $0.5 \mathrm{em}$ minus $0.4 \mathrm{emNew}$ York, NY, USA: John Wiley \& Sons, Inc., 1993.

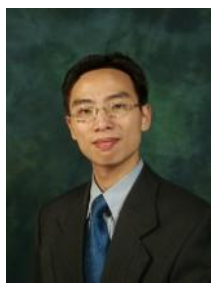

Joseph Chi-Ho So graduated from The University of Hong Kong with a Bachelor's degree in Electrical and Electronic Engineering. He then obtained a Master of Science from The Hong Kong Polytechnic University and $\mathrm{a} \mathrm{PhD}$ in Information Engineering from The Chinese University of Hong Kong. He had been working as an Internet Protocol Specialist at Pacific Century CyberWorks (PCCW) for several years, concentrating on Broadband Network Development, Network Management Systems, and Data Centre Services. Dr So also have been the Network Operation Centre Manager of a multinational internet infrastructure service provider. $\mathrm{He}$ is a Chartered Engineer. Dr So is particularly interested in the Peer-to-peer Networks, Internet and World Wide Web Technologies, Communication Networks, and Educational Technologies. Dr. So is a CENG, MHKIE, MIET and MIEEE.

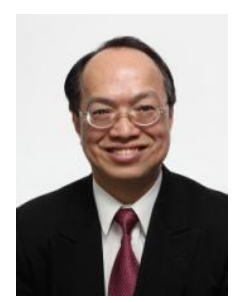

Prof. P. C. Wong is currently the Associate Vice-President (Development) of Hang Seng Management College. He is a renowned IT expert in Hong Kong. In 1998, he launched the Hong Kong Cyber Campus project to bring the Internet era to schools in Hong Kong. In 2004, he launched the VChina project, a video conferencing network linking hundreds of schools in HK, China, and overseas. He published widely and has received many awards, the IT Application Award in 1998, two US Patents and the IT Excellence Award in 1999, two Grand Linux Awards in 2003, and the 10th anniversary QEF Outstanding Project Award in 2008. In 2001, he received the Medal of Honor from Hong Kong for his contributions to the use of IT in education. Prof. Wong was a professor in the Chinese University of Hong Kong for twenty years before changing to Hang Seng Management College to become their Associate Vice President (Development). 